\title{
The Protein Modification and Degradation Pathways after Brain Ischemia
}

\author{
Tibor Kristian $^{1,2}$ (D) Bingren $\mathrm{Hu}^{1,2}$
}

Received: 19 September 2017 / Accepted: 21 September 2017 /Published online: 1 October 2017

(C) Springer Science+Business Media, LLC 2017

This Special Issue of Translational Stroke Research is focused on the protein modification and degradation pathways after brain ischemia and is a continuation of a previous special issue in this journal entitled: "The protein degradation pathways after brain ischemia" [1]. Articles in this current issue summarize recent developments and understanding of protein aggregation, modification, and degradation triggered by ischemic insult. This Special Issue seeks to reiterate that disruption in cellular protein metabolism remains an important aspect of ischemic brain injury and that mechanisms of acute neuronal cell death are closely related to those of neurodegenerative disease.

Few neurological conditions are as complex and devastating as ischemia-induced brain damage. Since metabolic processes are controlled and regulated by enzymes, disturbances in protein activity regulation and turnover significantly contribute to the underlying pathophysiologic mechanisms. Ischemic insult affects all cell types and practically every aspect of cellular metabolism. Therefore, the complex response of cells to ischemic insult cannot be explained by a single pathway or mechanism. However, this is an unresolved issue since changes in protein modification and cellular signaling pathways after brain ischemia still remains poorly understood. While brain ischemia certainly results in multiple disruptions

Tibor Kristian

tkristian@som.umaryland.edu

Bingren $\mathrm{Hu}$

bhu@som.umaryland.edu

1 Department of Anesthesiology, Shock Trauma and Anesthesiology Research Center, University of Maryland School of Medicine, Baltimore, MD 21201, USA

2 Veterans Affairs Maryland Health Center System, 10 North Greene Street, Baltimore, MD 21201, USA of cellular metabolic pathways, some may have a more significant impact on cell fate than others. To survive this pathologic environment, post-ischemic neurons must cope with repair and eliminate irreversibly damaged organelles and toxic materials by upregulation of cellular defense systems.

This special issue of Translational Stroke Research is focused on alterations in protein metabolism, degradation mechanisms, and post-translational modifications following brain ischemic insult. Each individual article highlights and discusses a specific issue related to the topic of post-ischemic alterations in cellular protein metabolism and its contribution to ischemic cell death.

$\mathrm{Hu}$ and colleagues discuss the pathophysiology of dysfunctional membrane trafficking mechanisms in neurons [2]. The authors hypothesize that dysfunction of a master protein that regulates membrane trafficking, $\mathrm{N}$-ethylmaleimide sensitive factor (NSF) ATPase, is a major reason leading to downstream pathophysiology of intracellular organelles interactions with Golgi fragmentation and late lysosomal damage. Following ischemia, NSF is deposited into the protein aggregates leading to its inactivation. This results in a cascade of events associated with disruption of the Golgi-endosome-lysosome pathway, release of cathepsin B (CTSB), and induction of mitochondrial outer membrane permeabilization [2]. Thus, this novel hypothesis suggests that NSF inactivation triggers massive release of CTSB leading to dysfunctional mitochondria and ultimately cell death. In the next article, Dong and colleagues present data that show a massive buildup of lateendosomes and fatal release of cathepsin B in CA1 neurons after transient forebrain ischemia [3]. Hochrainer in her sweeping review article addresses the significance of protein modifications with ubiquitin after cerebral ischemia and their role in mechanisms of brain injury. The review is critical in discussing current knowledge and controversies on protein ubiquitination, with emphasis on possible functional 
implications and development of neuroprotective strategies [4]. Klimova and colleagues provide a comprehensive review of post-translational modification of mitochondrial proteins with a focus on enzymes that control mitochondrial dynamics [5]. These modifications include SUMOylation, ubiquitination, phosphorylation, S-nitrosylation, acetylation, $O$-linked $N$-acetyl-glucosamine glycosylation, ADP-ribosylation, and proteolytic cleavage. Interestingly, one type of post-translational modification triggered under different stress conditions can lead to an opposite effect on mitochondrial dynamics. The authors hypothesize that this can be a result of interplay between several different modifications reflecting a complex regulatory mechanism of mitochondrial fusion and fission. Following ischemic insult there is an excessive mitochondrial fragmentation that is transient in cells within the ischemia resistant brain regions and permanent in cells destined to die. The authors emphasize that reversing the extensive mitochondrial fission and ameliorate ischemic brain damage will require a treatment strategy that will target several pathways that control individual posttranslational modifications. At last, Sun and colleagues focus on post-ischemic hypoperfusion and other pathologies shared between Alzheimer's disease and ischemia-induced neurodegeneration [6].

Together, these articles illustrate several important aspects of brain ischemia-reperfusion injury. The understanding of molecular mechanisms of acute brain injury is certain to impact future therapeutic efforts. Therefore, this Special Issue is timely in bringing together information pertinent to ischemia- induced protein modification and degradation pathways, and to disseminate these findings to foster further interest and research recognizing this multi-angled viewpoint.

\section{Compliance with Ethical Standards}

Conflict of Interest The authors declare that they have no conflict of interest.

Ethical Approval This article does not contain any studies with human participants or animals performed by any of the authors.

\section{References}

1. Degracia D, Hu B. Protein misfolding and organelle stress after brain ischemia. Transl Stroke Res. 2013 Dec;4(6):579-80. https://doi.org/ 10.1007/s12975-013-0302-1.

2. Yuan D, Liu Ch, Hu B. Dysfunction of membrane trafficking leads to ischemia-reperfusion injury after transient cerebral ischemia. Transl Stroke Res. 2017, in press.

3. Yuan D, Liu Ch, Hu B. Inactivation of NSF ATPase leads to cathepsin B release after brain ischemia. Transl Stroke Res. 2017, in press.

4. Hochrainer K. Protein modifications with ubiquitin as response to cerebral ischemia-reperfusion injury. Transl Stroke Res. 2017; in press

5. Klimova N, Long A, Kristian T. Significance of mitochondrial protein post-translational modifications in pathophysiology of brain injury. Transl Stroke Res. 2017, in press.

6. Dong S, Maniar S, Manole MD, Sun D. Cerebral hypoperfusion and other shared brain pathologies in ischemic stroke and Alzheimer's disease. Transl Stroke Res. 2017, in press. 